\title{
Schisandrin B, a Lignan from Schisandra chinensis Prevents Cerebral Oxidative Damage and Memory Decline Through Its Antioxidant Property
}

\author{
Tetsuya Konishi*, Vijayasree V. Giridharan \\ and Rajarajan A. Thandavarayan \\ Department of Functional and Analytical Food Sciences, Niigata University of Pharmacy \\ and Applied Life Sciences, Niigata, \\ Japan
}

\section{Introduction}

Increasing longevity over the coming decades is expected to cause a dramatic increase in the prevalence of dementia. The resources required to care for people with dementia will rise along with the prevalence. Healthcare systems are largely unprepared for the expects rise in prevalence and for the complex care many people with dementia require. It is important to note that prevention may not be "all or none". Current pharmaceutical treatment for dementia can only modestly improve symptoms and cannot cure or prevent dementia. As a result, prevention of dementia through identification and modification of risk facors is critical (Patrick McNamara, 2011)). However, rapidly growing evidence suggest that oxidative stress play a major role in the pathophysiology of neurodegenerative disease (Ali qureshi ali G Syed and Parvez SH, 2004).

Oxidative stress is the result of an imbalance in pro-oxidant/antioxidant homeostasis that leads to the generation of toxic reactive oxygen species (ROS). The brain is considered to be especially vulnerable towards oxidative stress due to several reasons: The brain is the highest utilization of inspired oxygen, the large amount of easily oxidizable polyunsaturated fatty acids, the abundance of redox-active transition metal ions, and the relative dearth of antioxidant defense systems. Free radicals are produced from a number of sources, among which are enzymatic, mitochondrial, and redox metal ion-derived sources. Hence, brain cells are continuously exposed to ROS generated by oxidative metabolism, and in certain pathological conditions, defense mechanisms against oxygen radicals may be weakened and/or overwhelmed (Butterfield DA, Stadtman ER, 1997).

Recent reports have established that the oxidative stress and damages are playing a role in the pathogenesis of a number of neurodegenerative diseases including Alzheimer's disease (AD), Parkinson disease (PD), corticobasal degeneration, Pick's disease and Alexander's disease (Gerst, Siedlak et al. 1999). Since these neurodegenerative diseases are the serious

${ }^{*}$ Corresponding Author 
factor decreasing quality of life in the longevity of society, the prevention of cerebral oxidative stress in an emergent of social task. (Konishi 2009). Many approaches have been reported such as the use of simple antioxidant molecules including antioxidant vitamins or dietary antioxidant to benefit neurodegeneration. (Srinivasan, Pandi-Perumal et al. 2005; Strimpakos and Sharma 2008; Sun, Wang et al. 2010) Among the various neurodegenerative disorders, the dementia is given importance and is focused in the present article.

Dementia is a brain disorder characterized by progressive memory loss and cognitive dysfunction, which occurs in mid to late life (McKhann, Drachman et al. 1984). Dementias and other severe cognitive dysfunction states pose a daunting challenge to existing medical management strategies. An integrative, early intervention approach seems warranted. Accumulating evidence suggests that nutritional and botanical therapies are attractive since they have proven degrees of efficacy and generally favorable benefit-to-risk profiles (Iriti M et al., 2010). Furthermore, several follow-up studies have reported a decreased risk of dementia assiciated with $\mathrm{AD}$ with increasing dietary or supplementary intake of antioxidants (Barberger-Gateau, Raffaitin et al. 2007). Thus antioxidant traditional herbal prescriptions was implicated as promised approach to prevent cerebral oxidative injury and prevent decline of brain function (Konishi T, 2009). Since many antioxidant ingredients have been identified in the component herbs of oriential medicine prescriptions, it is interesting to know the isolated antioxidant ingredient is active against cerebral oxidative stress as original herb or related formula. In the present article, we focus our attention onto Schisandrin B as a typical example of such herbal ingredient.

Schisandrin B (Sch B) is the major lignan with dibenzocyclooctadiene structure isolated from the fruit of Schisandra chinensis (FS) which is a major component of herb medicine belonging to Magnoliaceae family ( $\mathrm{Li}, \mathrm{Xu}$ et al. 2005) It is also one of three constituent herbs of famous traditional oriental medicine prescription, Shengmai san. Earlier, we have demonstrated the potential of Shengmai san to prevent cerebral oxidative damage and cerebral-ischemia injury in rat model (Xuejiang, Magara et al. 1999; Ichikawa, Wang et al. 2006). We also have reported quite recently the potential of Shengmai san in preventing scopolamine induced cerebral oxidative stress and memory dysfunction (Giridharan, Thandavarayan et al. 2011). Among the three component herbs, FS showed major contribution to the antioxidant activity of Shengmai san (Ichikawa H, 2003) and thus Sch B might be the major antioxidant ingredient characterizing antioxidant property of Shengmai san. In the present article, we discuss the potential of Sch B in preventing brain disorder especially in preventing cerebral oxidative stress and improving memory. It is our attempt to put forth the evidence for involvement of free radicals in pathophysiology of dementia and the potential benefit of treatment with antioxidants and radical scavengers by showing the role of dietary antioxidants Sch B in preventing oxidative stress induced cognitive disorders. We also put forth the behavioral and biochemical evidence for the potential of Sch B as a memory improving agent.

\section{Schisandrin B}

Sch B is the isolated component from the Schisandra chinensis.

Latin name: Fructus Schisandrae

Common name: Chinese magnoliavine fruit

Scientific Name: Schisandra chinensis, Schisandra sphenanthera

Chinese Name: $\mathrm{Wu}$ wei zi 
A

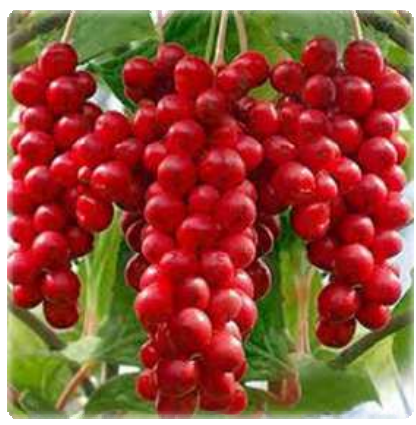

Schisandra chinensis
B

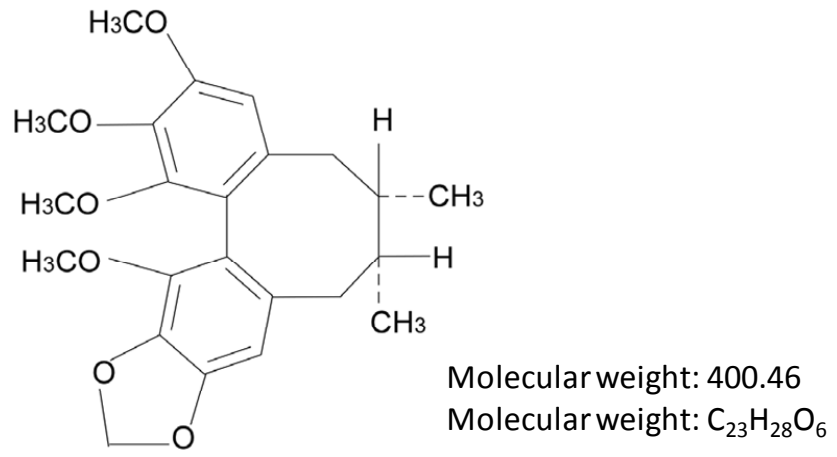

Schisandrin B

Fig. 1. A) Fruits of Schisandra chinensis; B) Structure of Sch B

The Chinese name of FS, Wuweizi is actually comprised of three Chinese words. The first word "Wu" means "five". The second word "Wei" means "taste" and the third word "Zi" refers to "seed". As the name of the herb suggests, FS is a seed with five tastes, which are sour, bitter, sweet, pungent and salty. Schisandra species grow in China, Japan, Eastern Russia, the Himalayas and Korea. FS traditionally used as astringent, to promote fluid production, to relieve pupil dilation, to relieve heat and arrest sweating and vomiting and to relieve diarrhea. The number of lignan isolated from FS includes schisandrin and its derivatives $\alpha_{-}^{-}, \beta_{-}^{-}, \gamma_{--}^{-} \delta_{-}^{-}, \varepsilon_{-}^{-}$ schisandrin, psedo- $\gamma$-schisandrin, deoxyschisandrin, neoschisandrin, schisandrol and others (Wang, Hu et al. 2008).

\section{The antioxidant potential of Sch B in various organs}

The isolated lignan from FS reported to possess the protective effects against various organs due to its strong antioxidant potential. The hepato-protective effect of Sch B against carbon tetra chloride toxicity was mediated by both enhancements of mitochondrial glutathione antioxidant status and heat shock proteins. (Zhu, Lin et al. 1999; Tang, Chiu et al. 2003). Further in in-vitro model Sch B elicits a glutathione antioxidant response and protects against apoptosis via the redox-sensitive ERK/Nrf2 pathway in AML12 heptocytes. (Leong, Chiu et al. 2011) Sch B treatment increases antioxidant status of the heart and improves cardiac function against the adiramycin, doxorubicin and ischemia/reperfusion induced cardiac dysfunction (Li, Pan et al. 2007; You, Pan et al. 2006; Chiu and Ko 2004). It has been reported that Sch B enhances renal mitochondrial antioxidant status and protects against gentamicin-induced nephro-toxicity in rats. (Chiu, Leung et al. 2008) Furthermore, renal failure induced by the acute oxidant mercuric chloride found to be decreased in Sch B treated rats.(Stacchiotti, Li Volti et al. 2011).

\subsection{Neuroprotective potential of Sch B}

Chen and coworkers have reported that long-term treatment with Sch B enhances mitochondrial antioxidant status, structural integrity against the cerebral ischemia/reperfusion injury in rat model. The cerebro-protection afforded by Sch B treatment 
was associated with increases in the levels and activity of mitochondrial antioxidant components (GSH, a-TOC, and Mn-SOD), as well as preservation of mitochondrial structural integrity. Structural integrity as indicated by the decrease in sensitivity to $\mathrm{Ca}^{2+}$ stimulated mitochondrial permeability transition in-vitro, was further evidenced by decrease in the extents of mitochondrial malondialdehyde (MDA) production, $\mathrm{Ca}^{2+}$ loading, and cytochrome $\mathrm{C}$ release (Chen, Chiu et al. 2008). Sch B also shown to have protection against L-glutamate induced neurotoxicity and the protection was associated with 1) an inhibition of the increase of intracellular $\left[\mathrm{Ca}^{2+}\right]$; 2) an improvement in the glutathione defense system, the level of glutathione, and the activity of glutathione peroxidase (GPx); and 3) an inhibition in the formation of cellular peroxide (Kim, Lee et al. 2004). Recently Sch B was reported to have protection against amyloid beta and homocysteine induced neurotoxicity in PC12 in-vitro system (Song, Lin et al.; Wang and Wang 2009). In addition we have also reported the potential of Sch B against scopolamine induced cerebral oxidative stress and memory dysfunction. (Giridharan, Thandavarayan et al. 2011). Of all the above reports states the neuroprotective potential of Sch B on the basis of its antioxidant property.

\section{Behavioral evidences}

\subsection{Passive avoidance task (PAT)}

We currently showed the neuroprotective effects of Sch B against experimental dementia induced by scopolamine and cisplatin (cDDP) (Giridharan, Thandavarayan et al., 2011). Passive avoidance behavior based on negative reinforcement was used to examine the long term memory (Giridharan, Thandavarayan et al. 2011). In this test, subjects learn to avoid an environment in which an aversive stimulus (such as a foot-shock) was previously delivered.

A

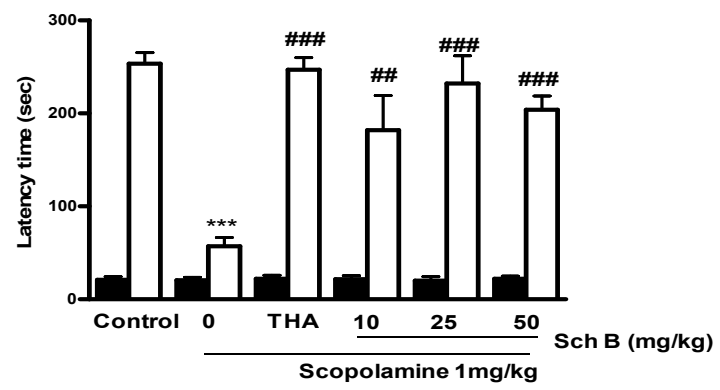

B

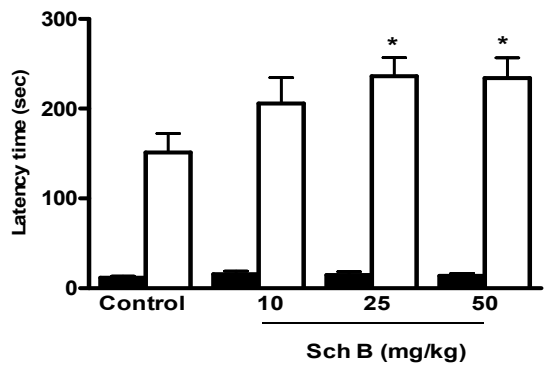

Acquisition

Retention

Fig. 2. A. Effects of Sch B on scopolamine-induced memory impairment in the PAT response in mice. For the study on the effect of Sch B on scopolamine-induced memory deficit model, mice were administered Sch B $(10,25$ and $50 \mathrm{mg} / \mathrm{kg})$ or THA $(10 \mathrm{mg} / \mathrm{kg}$, p.o., positive control) $1 \mathrm{~h}$ before the acquisition trial. Memory impairment was induced by scopolamine treatment $(1 \mathrm{mg} / \mathrm{kg}$, i.p.) and acquisition trials were carried out $30 \mathrm{~min}$ after scopolamine treatment. At $24 \mathrm{~h}$ after the acquisition trials, retention trials were carried out. Data represents mean \pm S.E.M $(\mathrm{n}=6) .{ }^{*} p<0.05,{ }^{* * *} p<0.001$, statistically different from control group. \#\#\# $p<0.001$, \#\# $p<0.01$ statistically different from scopolamine-treated group. 
The animals can freely explore the light and dark compartments of the chamber and a mild foot shock is delivered in one side of the compartment. Animals eventually learn to associate certain properties of the chamber with the foot shock. The latency to pass the gate in order to avoid the stimulus is used as an indicator of learning and memory. The passive avoidance task is useful for evaluating the effect of novel chemical entities on learning and memory as well as studying the mechanisms involved in cognition.

In the PAT, the anticholinergic agent scopolamine induced increase in step-through latency was finely inhibited by Sch B treatment. Sch B alone treated mice also found to have significant memory improving effect as showing Figure 2B. Sch B at the dose of $25 \mathrm{mg} / \mathrm{kg}$ recovered the memory level to $75.4 \%$ and thus the activity was comparable to tacrine (THA) $(76.9 \%)$ the standard drug used in the treatment for AD. (Giridharan, Thandavarayan et al. 2011). We have observed that shengmai san also found to inhibit scopolamine induced memory deficits in PAT model (Giridharan, Thandavarayan et al. 2011).

\subsection{Morris water maze test}

The acquisition and retention of a spatial navigation task is examined using a Morris Water Maze (Kumar, Seghal et al. 2006). The hippocampal formation plays an important role in memory and learning. The Morris Water Maze (MWM) is a test of spatial learning for rodents that relies on distal cues to navigate from started locations around the perimeter of an open swimming arena to locate a submerged escape platform. Spatial learning is assessed across repeated trials and reference memory is determined by preference for the platform area when the platform is absent (Vorhees and Williams 2006).

The memory enhancing potential of Sch B was also observed in the spatial memory task where Sch B treatment significantly decreased the escape latency and the result was comparable to that of THA. In the probe trail the time spent in the target quardrant was significantly improved the Sch B (Giridharan, Thandavarayan et al. 2011).

A

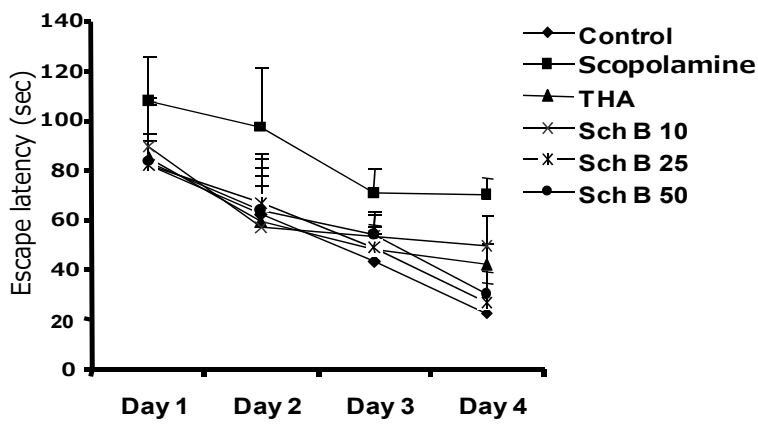




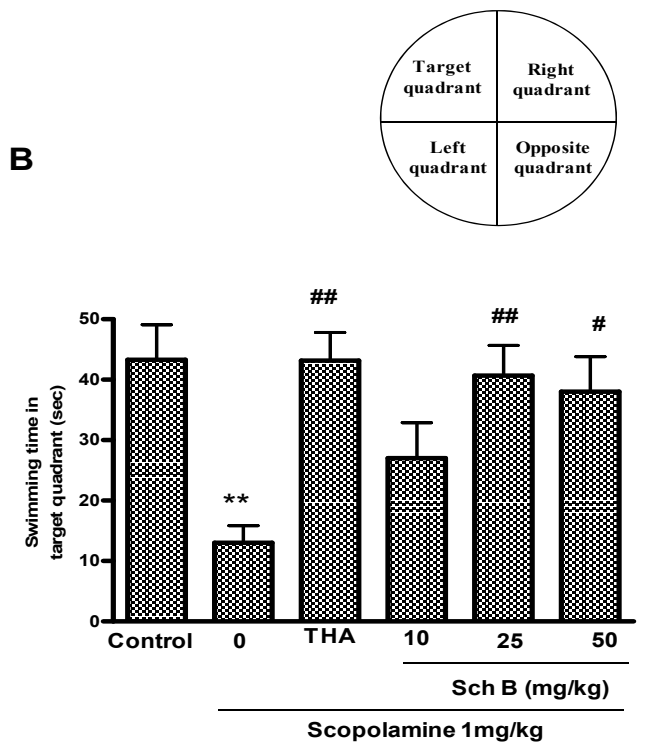

Fig. 3. Effect of Sch B on performance during training trial sessions (A) and probe trial sessions (B) of the MWM in scopolamine-induced memory deficit mice. At $1 \mathrm{~h}$ before the training trial session, Sch B (10,25 and $50 \mathrm{mg} / \mathrm{kg})$ or THA $(10 \mathrm{mg} / \mathrm{kg}$, p.o., positive control) was administered to mice. Memory impairment was induced by scopolamine treatment $(1$ $\mathrm{mg} / \mathrm{kg}$, i.p.) $30 \mathrm{~min}$ after Sch B or THA administration. Data represents mean \pm S.E.M $(\mathrm{n}=6)$. ${ }^{* *} p<0.01$, statistically different from control group. ${ }^{\# \#} p<0.01,{ }^{*} p<0.05$ statistically different from scopolamine-treated group.

\subsection{Elevated plus maze test (EPM)}

The elevated plus maze has been described as a simple method for assessing anxiety responses of rodents. There is great diversity in possible applications of the elevated plus maze. The elevated plus maze can be used as a behavioral assay to study the brain sites (e.g., limbic regions, hippocampus, amygdala, dorsal raphe nucleus, etc Furthermore, beyond its utility as a model to detect anxiolytic effects can also be used as a behavioral assay to study the brain sites (e.g., limbic regions, hippocampus, amygdala, dorsal raphe nucleus, etc.) and mechanisms (e.g., GABA, glutamate, serotonin, hypothalamic-pituitary-adrenal axis neuromodulators, etc.) underlying anxiety behavior. (Gonzalez and File 1997; Walf and Frye 2007). Briefly, rodents are placed in the intersection of the four arms of the elevated plus maze and their behavior is typically recorded for $5 \mathrm{~min}$. The behaviors that are typically recorded when rodents are in the elevated plus maze are the time spent and entries made on the open and closed arms.

Behavior in this task (i.e., activity in the open arms) reflects a conflict between the rodent's preference for protected areas (e.g., closed arms) and their innate motivation to explore novel environments. Anti-anxiety behavior (increased open arm time and/or open arm entries) can be determined simultaneously with a measure of spontaneous motor activity (total and/or closed arm entries). As shown in the figure treatment with Sch B at higher 
dose significantly increased the open arm entry, suggesting its anti-anxiety property (Giridharan, Thandavarayan et al 2011).

A

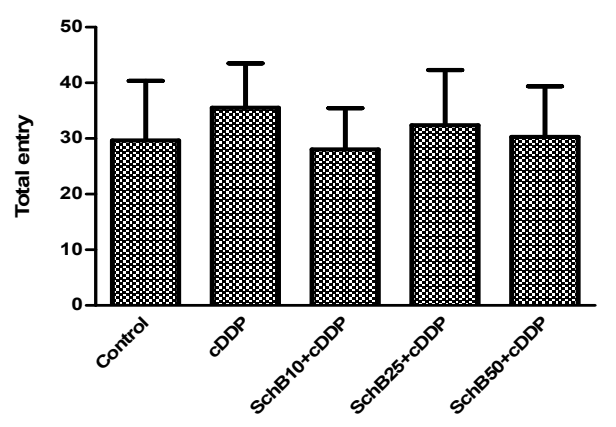

B

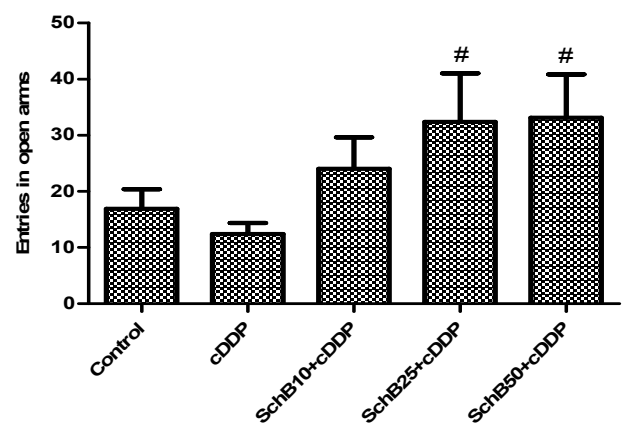

Fig. 4. Effect of Sch B (10, 25, and $50 \mathrm{mg} / \mathrm{kg})$ on the EPM task against cisplatin. (A) Total number of entries (B) Entries in open arms. Data are represented as the mean \pm S.E.M $(n=8)$. $\# p<0.05$, statistically different from cisplatin-treated group.

\section{Biochemical evidences}

\subsection{Cholinergic relationship with Sch B}

For a quarter of a century, the pathogenesis of $\mathrm{AD}$ associated dementia has been linked to a deficiency in the brain neurotransmitter acetylcholine (ACh). This was based on the observations of cholinergic system abnormalities leading to intellectual impairment. Subsequently, the 'cholinergic hypothesis' of AD gained considerable acceptance. It stated that a serious loss of cholinergic function in the central nervous system contributed to cognitive symptoms. Over the years, both evidence for and challenges to the relationship between $\mathrm{ACh}$ dysfunction and $\mathrm{AD}$ have been put forward, and acetylcholinesterase inhibitors (AChEIs) were introduced for the symptomatic treatment of AD. The prevailing view is that the efficacy of AChEIs is attained through their augmentation of AChmedicated nerve transmission. (Tabet 2006). 
Currently, the evidence was provided by us that Sch B act as a cholinesterase inhibitor by decreasing the levels of aceylcholinesterase (AChE) and improving the levels of ACh against scopolamine induced memory deficits animals (Giridharan, Thandavarayan et al. 2011).

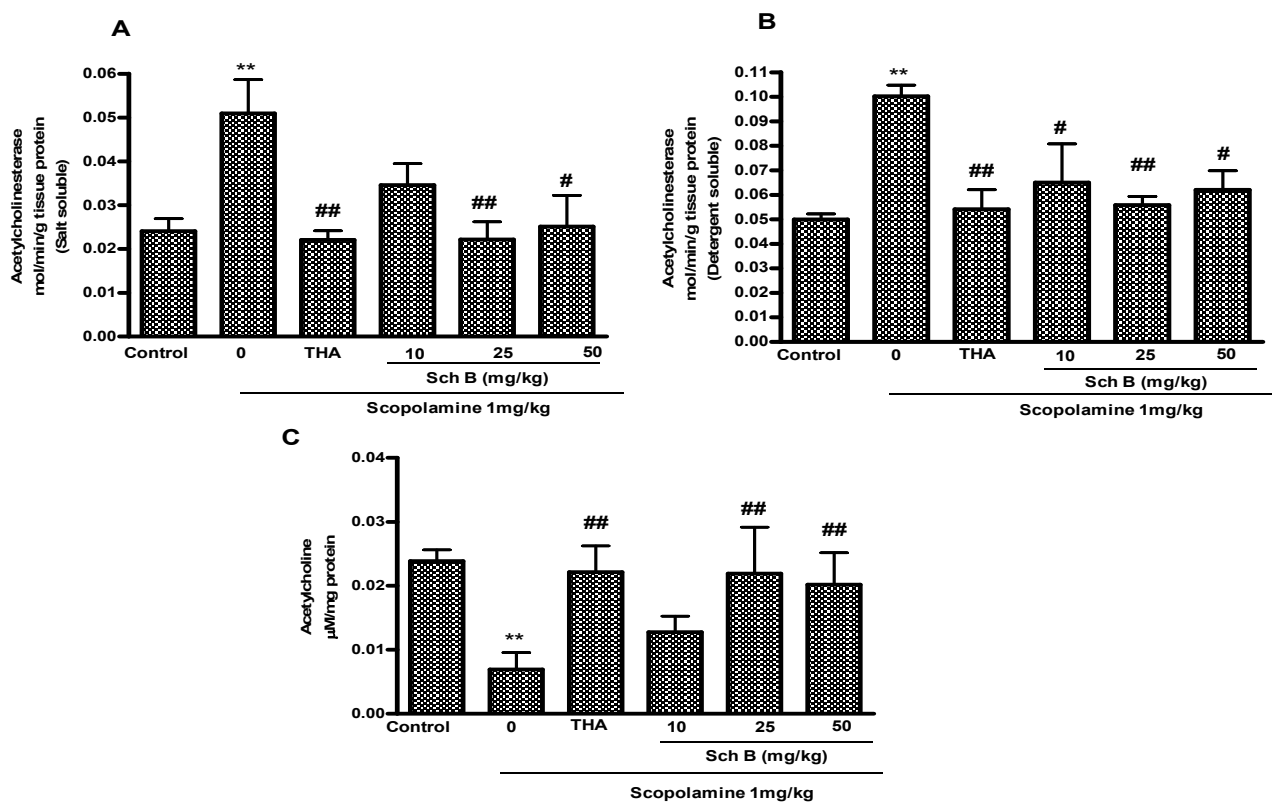

Fig. 5. The effect of Sch B $(10,25$ and $50 \mathrm{mg} / \mathrm{kg})$ administration for 7 days on AChE activity in SS fraction (A) and DS fraction (B) on ACh levels (C) of brain homogenate in scopolamineinduced memory deficit mice. Data represents mean \pm S.E.M $(n=6) .{ }^{* *} p<0.01$ statistically different from control group. ${ }^{\mathrm{p}}<0.05$, \#\# $p<0.01$, statistically different from scopolaminetreated group.

It is well documented that the AChE occurs in different molecular isoforms having differential localizations in neuronal cells. The two major isoforms are globular monomer (G1) and globular tetramer (G4) of the same monomer subunit. The G1 isoform is reported to present in the cytoplasm of neuronal cells, whereas the G4 isoform is predominantly membrane-bound (Massoulie, Pezzementi et al. 1993). In the present study, both forms were measured according to the method of Das et al (Das, Dikshit et al. 2005). Results showed that the AChE (G1 and G4 isoforms) levels in both salt soluble (SS) and detergent soluble (DS) fractions were significantly increased compared to normal control after scopolamine treatment but Sch B treatment reduced the level in both SS and DS fractions dosedependently. The percentage reduction of AChE activity in both SS and DS brain homogenates was $56.5 \%$ and $44.3 \%$, respectively, at $25 \mathrm{mg} / \mathrm{kg}$ of Sch B and the values are comparable to those of THA $(56.8 \%$ and $45.97 \%)$.

When direct inhibitory action of Sch B was examined on AChE activity in vitro, the IC50 values obtained was $>500 \mu \mathrm{M}$ that was far larger than the value of THA (approximately 2 $\mathrm{nM}$ ). Therefore, the inhibitory effect of Sch B may involve other mechanism than its direct inhibition of the enzyme. Further we analyzed the ACh levels in the brain homogenate of 
memory deficits mice. We observed that, ACh levels were significantly reduced in scopolamine-treated mice but treatment with Sch B $(25$ and $50 \mathrm{mg} / \mathrm{kg})$ increased the reduced ACh level as well as THA.

Altogether, our data suggest that the ameliorating effects of Sch B on memory deficit might involve the modulation of ACh level through an inhibition of enzyme. (Giridharan, Thandavarayan et al. 2011).

\section{Prevention of oxidative DNA damage by Sch B}

Oxidative DNA damage is an inevitable consequence of cellular metabolism, with a propensity for increased levels following toxic insult. Of the molecules subject to oxidative modification, DNA has received the greatest attention as the biomarkers of exposure and effect closest to validation. (Cooke, Evans et al. 2003; Evans, Dizdaroglu et al. 2004). Although ROS can attack a variety of biomolecules, DNA may be the primary target of the free radical damage that contributes to cellular degeneration and aging (Markesbery and Lovell 2006). Indeed, multiple studies show oxidative damage to DNA may be important in cancer and, because of its high oxygen consumption rate, may also be important in neuronal damage associated with aging and neurodegenerative diseases.(Lovell and Markesbery 2007).
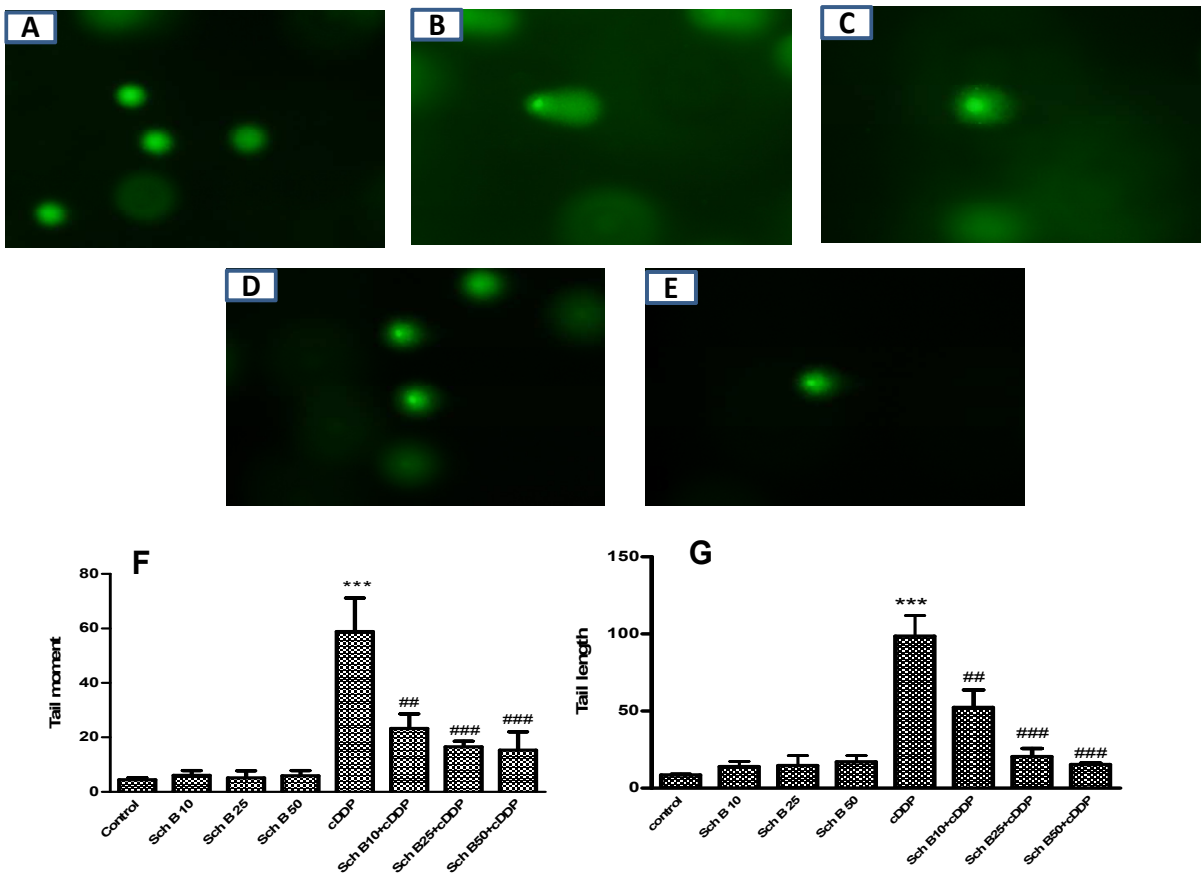

Fig. 6. Photomicrographs showing comets from forebrain stained with SYBR Green-II (A-E) Comet in a (A) normal cell (B) cisplatin-treated cell (C) Sch B 10+ cisplatin -treated cell (D) Sch B $25+$ cisplatin -treated cell, (E) Sch B 50+ cisplatin -treated cell (F) Tail moment (G) Tail length. $\mathrm{N}=4{ }^{* * *} p<0.01$ statistically different from control group. \#\#\# $p<0.001$, \#\# $p<0.01$ statistically different from cisplatine-treated group. 
The protective effect of Sch B was studied by the classical comet assay against chemotherapeutic agent cisplain -induced DNA damage in mouse brain (Fig.6) Treatment with Sch B effectively inhibited the cisplatin induced oxidative DNA damage as measured in terms of tail length and tail moment (Giridharan, Thandavarayan et al, 2011).

\section{The antioxidant potential of Sch B}

It is stated that along with increased oxidative damage, impaired antioxidant defenses have also been proposed to be prominent features of AD. Usually, the body produces different antioxidants (endogenous antioxidants) to neutralize free radicals and protect the body from different diseases lead by the oxidative injury. Exogenous antioxidants externally supplied to the body through food also plays important role to protect the body. The body has developed several endogenous antioxidant defense systems classified into two groups such as enzymatic and non enzymatic. The enzymatic defense system includes different endogenous enzymes like superoxide dismutase (SOD), catalase (CAT), glutathione peroxidase (GPx), glutathione reductase (GR) and non enzymatic defense system included small antioxidant molecules including vitamin $\mathrm{E}$, vitamin $\mathrm{C}$ and reduced glutathione (GSH) (Harris 1992).

The antioxidant system uses GSH, the most abundant non -protein thiol, which buffers free radicals in brain tissue. It eliminates $\mathrm{H}_{2} \mathrm{O}_{2}$ and organic peroxides by GPx coupled with GSH oxidation to glutathione disulfide (GSSG). GSH is regenerated by redox recycling, in which GSSG is reduced to GSH by GR with consumption of one NADPH. A reduction in level of

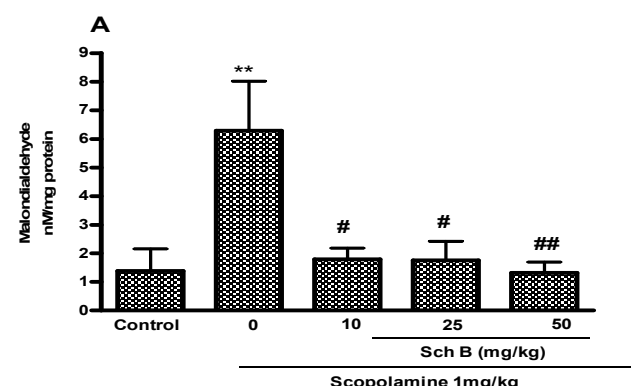

C

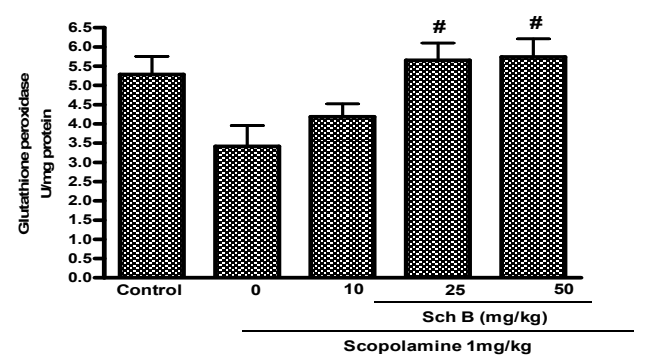

B

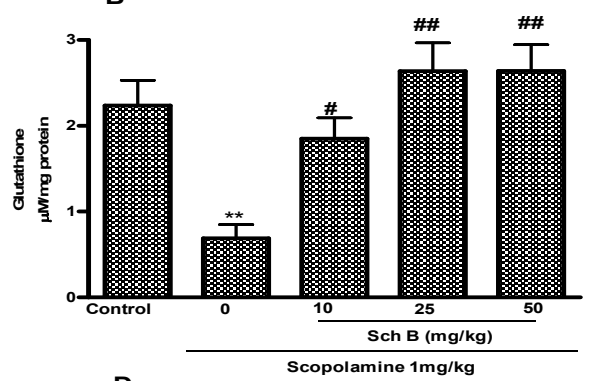

D

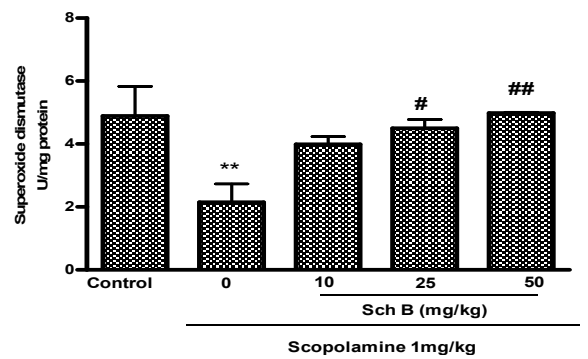

Fig. 7. Effects of acute Sch B $(10,25$ and $50 \mathrm{mg} / \mathrm{kg})$ treatment on the concentrations of MDA (A) and GSH (B) and activities of GPx (C) and SOD (D) in scopolamine-induced memory deficit mice. Data represents mean \pm S.E.M $(n=6) .{ }^{* *} p<0.01$, statistically different from control group. $\#$ $p<0.01, \# p<0.05$ statistically different from scopolamine-treated group. 
GSH may impair $\mathrm{H}_{2} \mathrm{O}_{2}$ clearance and promotes $\mathrm{OH}$ radical formation, one of the most toxic ROS to the brain leading to oxidative damage. The $\mathrm{OH}$ radical induces peroxidation of polyunsaturated fatty acids leading to the formation MDA, an end product of lipid peroxidation (Deshmukh, Sharma et al. 2009). Interestingly, SOD mimics have come to the forefront of antioxidative therapeutics of neurodegenerative disease (Pong 2003).

We have evaluated the antioxidant potential of Sch B against scopolamine and cisplatin induced cerebral oxidative stress. Treatment with Sch B significantly increased the levels of antioxidant enzymes such as GPx and SOD, and cellular GSH levels with parallel decrease in lipid peroxidation levels (Giridharan, Thandavarayan et al, 2011).

\section{Conclusion}

Oxidative stress is a ubiquitously observed hallmark of neurodegenerative disorders. Neuronal cell dysfunction and cell death due to oxidative stress may causally contribute to the pathogenesis of progressive neurodegenerative disorders, such as AD and PD, as well as acute syndromes of neurodegeneration, such as ischemic and haemorrhagic stroke. Neuroprotective antioxidants are considered a promising approach to slow the progression and limit the extent of neuronal cell loss in these disorders. The clinical evidence demonstrating that antioxidant compounds can act as protective drugs in neurodegenerative disease (Guglielmotto, Giliberto et al.) Recently, cholinesterase inhibitors hybrids such as THA-melatonin developed for the treatment of AD. As AD is considered as multi complex disease with various biochemical targets, multi target-directed ligand strategy is a logical approach for designing a suitable therapy.(Fernandez-Bachiller, Perez et al. 2009; Leon and Marco-Contelles 2011). In the present article, we provided evidence for the multi-factorial role of nutritional antioxidants Sch B which behaves as neuro-protective agent, anti-cholinergic agent, and also as potential antioxidants. Further studies are needed to know more precise molecular mechanism of Sch B function as neuroprotectant.

\section{Acknowledgement}

This study was supported by a grant to TK from the Promotion and Mutual Aid Corporation for Private Schools. The authors thank the Rotary Yoneyama Scholarship Association for the financial assistance to VVG.

\section{References}

Ali qureshi ali Syed G and Parvez SH; Oxidative Stress and Neurodegenerative Disorders 2004 Elsevier, role of selenium, iron, copper and zinc in parkinsonism edited By S. Hasan Parvez

Barberger-Gateau, P., C. Raffaitin, et al. (2007). "Dietary patterns and risk of dementia: the Three-City cohort study." Neurology 69(20): 1921-30.

Chen, N., P. Y. Chiu, et al. (2008). "Schisandrin B enhances cerebral mitochondrial antioxidant status and structural integrity, and protects against cerebral ischemia/reperfusion injury in rats." Biol Pharm Bull 31(7): 1387-91.

Chiu, P. Y. and K. M. Ko (2004). "Schisandrin B protects myocardial ischemia-reperfusion injury partly by inducing Hsp25 and Hsp70 expression in rats." Mol Cell Biochem 266(1-2): 139-44. 
Chiu, P. Y., H. Y. Leung, et al. (2008). "Schisandrin B Enhances Renal Mitochondrial Antioxidant Status, Functional and Structural Integrity, and Protects against Gentamicin-Induced Nephrotoxicity in Rats." Biol Pharm Bull 31(4): 602-5.

Cooke, M. S., M. D. Evans, et al. (2003). "Oxidative DNA damage: mechanisms, mutation, and disease." FASEB J 17(10): 1195-214.

Das, A., M. Dikshit, et al. (2005). "Role of molecular isoforms of acetylcholinesterase in learning and memory functions." Pharmacol Biochem Behav 81(1): 89-99.

Deshmukh, R., V. Sharma, et al. (2009). "Amelioration of intracerebroventricular streptozotocin induced cognitive dysfunction and oxidative stress by vinpocetine -a PDE1 inhibitor." Eur J Pharmacol 620(1-3): 49-56.

Evans, M. D., M. Dizdaroglu, et al. (2004). "Oxidative DNA damage and disease: induction, repair and significance." Mutat Res 567(1): 1-61.

Fernandez-Bachiller, M. I., C. Perez, et al. (2009). "Tacrine-melatonin hybrids as multifunctional agents for Alzheimer's disease, with cholinergic, antioxidant, and neuroprotective properties." ChemMedChem 4(5): 828-41.

Gerst, J. L., S. L. Siedlak, et al. (1999). "Role of oxidative stress in frontotemporal dementia." Dement Geriatr Cogn Disord 10 Suppl 1: 85-7.

Giridharan, V. V., R. A. Thandavarayan, et al. (2011). "Effect of Shengmai-san on cognitive performance and cerebral oxidative damage in BALB/c mice." J Med Food 14(6): 601-9.

Giridharan, V. V., R. A. Thandavarayan, et al. (2011). "Ocimum sanctum Linn. Leaf Extracts Inhibit Acetylcholinesterase and Improve Cognition in Rats with Experimentally Induced Dementia." J Med Food 14(9): 912-9.

Giridharan, V. V., R. A. Thandavarayan, et al. (2011). "Prevention of scopolamine-induced memory deficits by schisandrin B, an antioxidant lignan from Schisandra chinensis in mice." Free Radic Res 45(8): 950-8.

Giridharan, V. V., R. A. Thandavarayan, et al. (2011). " "Schisandrin B attenuates cisplatininduced oxidative stress, genotoxicity and neurotoxicity through modulating NFкB pathway in mice." Free Radic Res. 2011.

Gonzalez, L. E. and S. E. File (1997). "A five minute experience in the elevated plus-maze alters the state of the benzodiazepine receptor in the dorsal raphe nucleus." $J$ Neurosci 17(4): 1505-11.

Guglielmotto, M., L. Giliberto, et al. "Oxidative stress mediates the pathogenic effect of different Alzheimer's disease risk factors." Front Aging Neurosci 2: 3.

Harris, E. D. (1992). "Regulation of antioxidant enzymes." J Nutr 122(3 Suppl): 625-6.

Ichikawa, H., L. Wang, et al. (2006). "Prevention of cerebral oxidative injury by postischemic intravenous administration of Shengmai San." Am J Chin Med 34(4): 591600.

Kim, S. R., M. K. Lee, et al. (2004). "Dibenzocyclooctadiene lignans from Schisandra chinensis protect primary cultures of rat cortical cells from glutamate-induced toxicity." J Neurosci Res 76(3): 397-405.

Konishi, T. (2009). "Brain oxidative stress as basic target of antioxidant traditional oriental medicines." Neurochem Res 34(4): 711-6.

Kumar, A., N. Seghal, et al. (2006). "Differential effects of cyclooxygenase inhibitors on intracerebroventricular colchicine-induced dysfunction and oxidative stress in rats." Eur J Pharmacol 551(1-3): 58-66. 
Leon, R. and J. Marco-Contelles (2011). "A step further towards multitarget drugs for Alzheimer and neuronal vascular diseases: targeting the cholinergic system, amyloid-beta aggregation and $\mathrm{Ca}(2+)$ dyshomeostasis." Curr Med Chem 18(4): 55276.

Leong, P. K., P. Y. Chiu, et al. (2011). "Schisandrin B elicits a glutathione antioxidant response and protects against apoptosis via the redox-sensitive ERK/Nrf2 pathway in AML12 hepatocytes." Free Radic Res 45(4): 483-95.

Li, L., Q. Pan, et al. (2007). "Schisandrin B prevents doxorubicin-induced cardiotoxicity via enhancing glutathione redox cycling." Clin Cancer Res 13(22 Pt 1): 6753-60.

Li, Y., C. Xu, et al. (2005). "In vitro anti-Helicobacter pylori action of 30 Chinese herbal medicines used to treat ulcer diseases." J Ethnopharmacol 98(3): 329-33.

Lovell, M. A. and W. R. Markesbery (2007). "Oxidative DNA damage in mild cognitive impairment and late-stage Alzheimer's disease." Nucleic Acids Res 35(22): 7497-504.

Markesbery, W. R. and M. A. Lovell (2006). "DNA oxidation in Alzheimer's disease." Antioxid Redox Signal 8(11-12): 2039-45.

Massoulie, J., L. Pezzementi, et al. (1993). "Molecular and cellular biology of cholinesterases." Prog Neurobiol 41(1): 31-91.

McKhann, G., D. Drachman, et al. (1984). "Clinical diagnosis of Alzheimer's disease: report of the NINCDS-ADRDA Work Group under the auspices of Department of Health and Human Services Task Force on Alzheimer's Disease." Neurology 34(7): 939-44.

Pong, K. (2003). "Oxidative stress in neurodegenerative diseases: therapeutic implications for superoxide dismutase mimetics." Expert Opin Biol Ther 3(1): 127-39.

Song, J. X., X. Lin, et al. "Protective effects of dibenzocyclooctadiene lignans from Schisandra chinensis against beta-amyloid and homocysteine neurotoxicity in PC12 cells." Phytother Res 25(3): 435-43.

Srinivasan, V., S. R. Pandi-Perumal, et al. (2005). "Role of melatonin in neurodegenerative diseases." Neurotox Res 7(4): 293-318.

Stacchiotti, A., G. Li Volti, et al. (2011). "Different role of Schisandrin B on mercury-induced renal damage in vivo and in vitro." Toxicology 286(1-3): 48-57.

Strimpakos, A. S. and R. A. Sharma (2008). "Curcumin: preventive and therapeutic properties in laboratory studies and clinical trials." Antioxid Redox Signal 10(3): 51145.

Sun, A. Y., Q. Wang, et al. (2010). "Resveratrol as a therapeutic agent for neurodegenerative diseases." Mol Neurobiol 41(2-3): 375-83.

Tabet, N. (2006). "Acetylcholinesterase inhibitors for Alzheimer's disease: antiinflammatories in acetylcholine clothing!" Age Ageing 35(4): 336-8.

Tang, M. H., P. Y. Chiu, et al. (2003). "Hepatoprotective action of schisandrin B against carbon tetrachloride toxicity was mediated by both enhancement of mitochondrial glutathione status and induction of heat shock proteins in mice." Biofactors 19(1-2): 33-42.

Vorhees, C. V. and M. T. Williams (2006). "Morris water maze: procedures for assessing spatial and related forms of learning and memory." Nat Protoc 1(2): 848-58.

Walf, A. A. and C. A. Frye (2007). "The use of the elevated plus maze as an assay of anxietyrelated behavior in rodents." Nat Protoc 2(2): 322-8.

Wang, B. and X. M. Wang (2009). "Schisandrin B protects rat cortical neurons against Abeta1-42-induced neurotoxicity." Pharmazie 64(7): 450-4. 
Wang, B. L., J. P. Hu, et al. (2008). "Simultaneous quantification of four active schisandra lignans from a traditional Chinese medicine Schisandra chinensis(Wuweizi) in rat plasma using liquid chromatography/mass spectrometry." J Chromatogr B Analyt Technol Biomed Life Sci 865(1-2): 114-20.

Xuejiang, W., T. Magara, et al. (1999). "Prevention and repair of cerebral ischemiareperfusion injury by Chinese herbal medicine, shengmai san, in rats." Free Radic Res 31(5): 449-55.

You, J. S., T. L. Pan, et al. (2006). "Schisandra chinensis protects against adriamycin-induced cardiotoxicity in rats." Chang Gung Med J 29(1): 63-70.

Zhu, M., K. F. Lin, et al. (1999). "Evaluation of the protective effects of Schisandra chinensis on Phase I drug metabolism using a CCl4 intoxication model." J Ethnopharmacol 67(1): 61-8. 


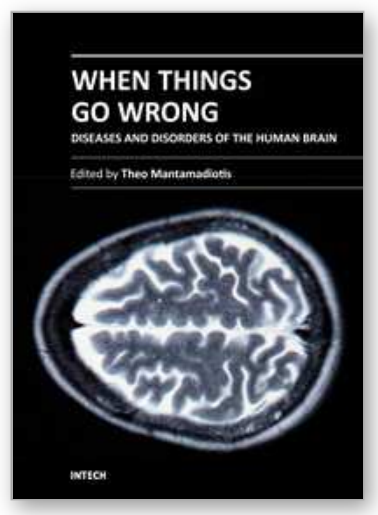

\author{
When Things Go Wrong - Diseases and Disorders of the Human \\ Brain \\ Edited by Dr. Theo Mantamadiotis
}

ISBN 978-953-51-0111-6

Hard cover, 238 pages

Publisher InTech

Published online 29, February, 2012

Published in print edition February, 2012

In this book we have experts writing on various neuroscience topics ranging from mental illness, syndromes, compulsive disorders, brain cancer and advances in therapies and imaging techniques. Although diverse, the topics provide an overview of an array of diseases and their underlying causes, as well as advances in the treatment of these ailments. This book includes three chapters dedicated to neurodegenerative diseases, undoubtedly a group of diseases of huge socio-economic importance due to the number of people currently suffering from this type of disease but also the prediction of a huge increase in the number of people becoming afflicted. The book also includes a chapter on the molecular and cellular aspects of brain cancer, a disease which is still amongst the least treatable of cancers.

\title{
How to reference
}

In order to correctly reference this scholarly work, feel free to copy and paste the following:

Tetsuya Konishi, Vijayasree V. Giridharan and Rajarajan A. Thandavarayan (2012). Schisandrin B, a Lignan from Schisandra chinensis Prevents Cerebral Oxidative Damage and Memory Decline Through Its Antioxidant Property, When Things Go Wrong - Diseases and Disorders of the Human Brain, Dr. Theo Mantamadiotis (Ed.), ISBN: 978-953-51-0111-6, InTech, Available from: http://www.intechopen.com/books/when-things-gowrong-diseases-and-disorders-of-the-human-brain/schisandrin-b-a-lignan-from-schisandra-chinensisprevents-cerebral-oxidative-damage-and-memory-decli

\section{INTECH}

open science | open minds

\section{InTech Europe}

University Campus STeP Ri

Slavka Krautzeka 83/A

51000 Rijeka, Croatia

Phone: +385 (51) 770447

Fax: +385 (51) 686166

www.intechopen.com

\section{InTech China}

Unit 405, Office Block, Hotel Equatorial Shanghai

No.65, Yan An Road (West), Shanghai, 200040, China

中国上海市延安西路65号上海国际贵都大饭店办公楼 405 单元

Phone: +86-21-62489820

Fax: $+86-21-62489821$ 
(C) 2012 The Author(s). Licensee IntechOpen. This is an open access article distributed under the terms of the Creative Commons Attribution 3.0 License, which permits unrestricted use, distribution, and reproduction in any medium, provided the original work is properly cited. 